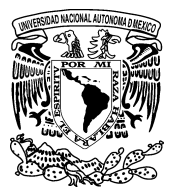

\title{
Sucesión y su relación con endeudamiento y desempeño en empresas familiares
}

\author{
Succession and its relationship with debt and performance \\ in family business
}

\section{Juan Manuel San Martín Reyna* y Jorge Alberto Durán Encalada}

Universidad de las Américas Puebla, Centro de Investigación de Empresas Familiares, México

Recibido el 27 de junio de 2014; aceptado el 7 de marzo de 2015

Disponible en Internet el 26 de octubre de 2015

\section{Resumen}

Las empresas familiares, además de tomar decisiones respecto a los problemas que les afectan, como todas las empresas, deben ser capaces de gestionar de forma adecuada la relación empresa-familia, evaluando las dinámicas que rigen la conducta de fundadores, próximas generaciones, familia, accionistas y empresa. Por tanto, es importante desarrollar habilidades que les permitan identificar y resolver las dificultades que planteen estas dinámicas y adoptar estrategias para fomentar su crecimiento, transferir el poder y el control, buscando generar valor para la compañía. Es por ello que este trabajo analiza el efecto de la sucesión en la estructura financiera y de capital de las empresas familiares. Basados en una muestra de empresas públicas mexicanas durante los periodos 2005-2011, encontramos que los efectos de la sucesión sobre el desempeño financiero de estas compañías es diferente al efecto que ejerce sobre ese desempeño una tercera generación al frente de la empresa, por lo que empresas familiares de segunda generación se caracterizan por mostrar una mayor aversión al riesgo en cuanto a endeudamiento, y una actitud más conservadora en comparación con empresas familiares de tercera generación, caracterizadas por una mayor preferencia por la financiación a través de la deuda, orientadas hacia una mayor creación de valor para la compañía.

Derechos Reservados ( 2015 Universidad Nacional Autónoma de México, Facultad de Contaduría y Administración. Este es un artículo de acceso abierto distribuido bajo los términos de la Licencia Creative Commons CC BY-NC-ND 4.0.

Palabras clave: Sucesión; Endeudamiento; Creación de valor; Empresa familiar

\footnotetext{
* Autor para correspondencia.

Correo electrónico: juanm.sanmartin@udlap.mx (J.M. San Martín Reyna).

La revisión por pares es responsabilidad de la Universidad Nacional Autónoma de México.
} 


\begin{abstract}
In addition to making decisions about issues that affect all companies, family business must be able to adequately manage the company-family relationship through an examination of the dynamics that involve founders, successive generations, shareholders and the firm. It is therefore important to develop skills to identify and resolve difficulties raised by these relationships, and adopt strategies to promote growth, share power and control, seeking to create value for the company. Thus why this paper analyzes the effect of succession on the financial and capital structure of family firms. Based on a sample of Mexican public companies during the 2005-2011 period, we find that the effects of succession on financial performance varies depending on the generation that leads the company. We find that second-generation family businesses are characterized by greater risk aversion to indebtness and are more conservative compared with third-generation family business, characterized by a greater preference for financing through debt.
\end{abstract}

All Rights Reserved (c) 2015 Universidad Nacional Autónoma de México, Facultad de Contaduría y Administración. This is an open access item distributed under the Creative Commons CC License BY-NC-ND 4.0.

Keywords: Succession; Debt; Firm value; Family business

\title{
Introducción
}

La investigación sobre empresas familiares se ha desarrollado de forma importante sobre todo a partir de la década de los 70, siendo uno de los tópicos más analizados el de la sucesión. El porqué la literatura se concentra tanto en este tema no es un misterio, las cifras fundamentan esta obsesión, ya que, de acuerdo a estas, pareciera que la principal causa de mortandad en las empresas familiares se encuentra en el proceso sucesorio al argumentarse que el $70 \%$ de los negocios familiares desaparecen después de la muerte del fundador, y solo entre el $9 \%$ y el $15 \%$ consiguen llegar a una tercera generación (Birley, 1986; Ward, 1997, Kets de Vries, 1993). En nuestro país las cifras son más escalofriantes, ya que con el impacto tan fuerte que estas empresas tienen en nuestro sistema económico, solo 3 de cada 10 empresas familiares logran sobrevivir a la segunda generación de acuerdo con entidades gubernamentales, como es la Secretaría de Economía (2014). Es decir, hablamos de empresas que contribuyen con casi el 70\% del empleo en el país y de las cuales solo una tercera parte sobrevivirá al relevo generacional. Como podemos apreciar, estos datos ponen de manifiesto que uno de los problemas más difíciles por los que atraviesan las empresas familiares es sin lugar a dudas la transmisión de la empresa a una siguiente generación.

No obstante, la sucesión es considerada un momento crucial e ineludible para la empresa y para la familia, ya que significa no solamente un paso necesario para la continuidad de la primera, sino para la seguridad de la segunda y la estabilidad de ambas (Trevinyo-Rodríguez, 2010). De acuerdo con Zúñiga y Sacristán (2009), el proceso de sucesión, junto con la profesionalización, son 2 de los principales desafíos a los que se enfrentan las empresas familiares para lograr ser más competitivas.

Muchos de los estudios previos sobre el tema se concentran en el hecho de que los problemas a que se enfrentará la compañía están en si el proceso de sucesión se da de forma armónica, logrando evitar ese salto, muchas veces abrupto, de pasar la estafeta a las nuevas generaciones. No obstante, muchas veces los problemas pueden darse una vez que se ha dado el relevo generacional, aspectos como la creación de valor en la empresa y la financiación son uno de los temas más recurrentes en 
la literatura. Además, es importante tener en cuenta que en el cambio generacional del fundador a la segunda generación (consorcio de hermanos), los problemas a los que se enfrenta la empresa pueden ser diferentes al cambio generacional que se da de la segunda generación en adelante (consorcio de primos), por lo que la propiedad, la aversión al riesgo y la toma de decisiones no son las mismas en un proceso que en otro. Estos procesos de sucesión a través de las generaciones enfrentan a la familia y a la empresa con una compleja serie de opciones. Si bien cada opción incluye diferentes oportunidades y riesgos, es importante analizar cuáles son los efectos que esta tiene sobre el desempeño financiero de la compañía.

En este sentido, diferentes estudios han encontrado evidencia de cambios en el desempeño financiero de las empresas familiares una vez que el proceso de sucesión ha tenido lugar en la compañía. Sin embargo, ha habido gran ambigüedad entre los resultados alcanzados por diferentes investigadores, derivada fundamentalmente de que las muestras de estudio provienen de diversos países y, por ende, de diversos ambientes de negocios.

Sin embargo, una de las principales limitaciones en los estudios realizados hasta ahora es que muchos de los trabajos han analizado esta relación entre sucesión en la empresa familiar y el desempeño financiero basados en datos de corte transversal en su mayoría (Bennedsen, Nielsen, Perez-Gonzalez y Wolfenzon, 2007; Blanco-Mazagatos, de Quevedo-Puente y Castrillo, 2007; Pérez-González, 2006), por lo que nosotros buscamos ampliar la base de datos utilizando técnicas como la de datos de panel que nos permitan capturar de mejor forma los cambios en el desempeño de una empresa familiar a raíz del cambio generacional a lo largo del tiempo. Por tanto, nuestro principal objetivo es analizar el efecto que el cambio generacional en la empresa tiene sobre la estructura financiera de la empresa (niveles de endeudamiento), así como también sus efectos sobre la creación de valor de la compañía (performance), y si estos efectos son diferentes dependiendo de si la transferencia generacional es de primera a segunda generación o de segunda a tercera generación (Davis y Harveston, 1999; Schulze, Lubatkin y Dino, 2003; Amit y Villalonga, 2006; Molly, Leveren y Deloof, 2010). Aunque el estudio sobre la empresa familiar ha sido abordado a través de distintas perspectivas, como la Teoría de los costos de transacción, la Teoría de juegos, la Teoría institucional, la Teoría de los recursos y capacidades, Teoría general de sistemas, entre otras, en este trabajo, basándonos en Molly et al. (2010), tomamos como fundamento la Teoría de la agencia (Jensen y Meckling, 1976) y del estancamiento (Miller, Le Breton-Miller y Scholnick, 2008).

Para ello, el artículo se estructura de la siguiente forma: en la siguiente sección se hace una revisión de la literatura sobre el impacto de la sucesión en la estructura financiera y el desempeño de la empresa familiar y se formulan las hipótesis. En la tercera sección se detalla la parte metodológica del trabajo. En la cuarta sección se presentan los resultados obtenidos y, finalmente, la última sección presenta la conclusión del trabajo, sus limitaciones y futuras líneas de investigación

\section{Revisión de la literatura}

Muchos estudios sobre sucesión toman como referencia la perspectiva de la agencia desarrollada por Jensen y Meckling (1976) basada en las asimetrías de información que se generan entre los directivos, accionistas y acreedores. Sin embargo, aunque muchos de estos estudios argumentan una menor exposición a los costos de agencia por parte de las empresas familiares (por ejemplo Daily y Dollinger, 1992; Fama y Jensen, 1983; Shleifer y Vishny, 1986; Casson, 1999; Chami, 1999; Castrillo y San-Martín, 2007) es importante señalar que estos problemas pueden no solo surgir, sino agravarse una vez que se da el proceso de sucesión dentro de la empresa. En este sentido, diferentes autores argumentan que muchos de estos problemas surgen debido a los posibles conflictos familiares desde el momento en que una siguiente generación se hace cargo de 
la dirección y toma las riendas de la compañía (Davis y Harveston, 1999; Smith y Amoako-Adu, 1999; Molly et al., 2010).

Por otra parte, autores como Miller et al. (2008) introducen la perspectiva del estancamiento para analizar la naturaleza de las empresas familiares. Esta perspectiva combina diferentes reflexiones sobre las empresas familiares, enfatizando la naturaleza conservadora de este tipo de empresas, ya que de acuerdo con Miller et al. (2008) la perspectiva de estancamiento puede manifestarse de varias maneras, como puede ser por la falta de recursos financieros y de capacidad de gestión, la aversión al riesgo y, por ende, una posible actitud más conservadora, lo que genera que se desarrolle una empresa familiar cuyos fundamentos pudiesen ser «débiles» en cierta medida. Así pues, la perspectiva del estancamiento de Miller et al. (2008) y la teoría de la agencia de Jensen y Meckling (1976) pueden ser particularmente relevantes para explicar el comportamiento de las empresas familiares que se encuentran en un proceso de cambio generacional.

\section{Sucesión y endeudamiento}

Los trabajos realizados sobre el impacto que la sucesión tiene sobre la estructura financiera de las empresas familiares se basan principalmente en datos de corte transversal, y los resultados obtenidos han sido diferentes (Molly et al., 2010). Por una parte, algunos estudios han encontrado un efecto positivo sobre los niveles de endeudamiento una vez que el relevo generacional se ha dado en la compañía, mientras que otros han encontrado una relación negativa entre sucesión y endeudamiento. Estos resultados han sido explicados tanto por la capacidad como por la disposición a endeudarse por parte de las empresas familiares que han atravesado por un cambio generacional.

En cuanto a este último (disposición al endeudamiento), algunos autores argumentan que cuando las empresas familiares pasan de una generación a otra se vuelven más reacias a endeudarse debido a que no quieren asumir riesgos. En este sentido, autores como Kaye y Hamilton (2004) establecen que las empresas familiares que han enfrentado un cambio generacional se encuentran más orientadas hacia la familia, ya que los descendientes suelen tener una menor disposición a tomar riesgos en comparación con sus padres y tienen una mayor preferencia por conservar la riqueza, en lugar de una mayor creación de la misma, por lo que tratan de mantener una estructura financiera conservadora. En este sentido Reid, Dunn, Cromie y Adams (1999) argumentan que este tipo de negocios familiares son más reacios a utilizar fuentes de financiación externas, por considerarlas «riesgosas» para la familia, debido a que podría diluir el control por parte de esta. De acuerdo con los autores, en la familia impera el deseo de transferir una empresa sana a través de diferentes generaciones, salvaguardando así el nombre de la familia y la «obra» creada por el fundador, lo que es compatible con la teoría del estancamiento (Miller y Le Breton-Miller, 2005).

Por su parte Anderson, Mansi y Reeb (2003), basados en la teoría de la agencia, consideran las asimetrías de información entre los acreedores y los accionistas, argumentando que los primeros pueden considerar la sucesión del fundador a la siguiente generación como nociva para sus intereses, debido a que los descendientes pueden estar menos cualificados para dirigir la empresa. Autores como Davis y Harveston (1999); Paul (1996) y Schwass (2005) señalan que el proceso de sucesión suele aumentar también el número de familiares involucrados en el negocio, por lo que al haber un mayor número de accionistas de la familia (tanto activos como pasivos) puede dar lugar a conflictos dentro de la propia familia que pueden impactar en el negocio no solo desde el aspecto de propiedad, sino también desde la toma de decisiones en elementos fundamentales, como es definir las tasas de reinversión de utilidades o el monto del pago de dividendos, por ejemplo, lo que puede terminar afectando el desempeño financiero de la 
compañía. Por lo tanto, los acreedores pueden estar menos dispuestos a proporcionar financiación a las empresas familiares que hayan enfrentado el cambio generacional. Sin embargo, autores como Schulze et al. (2003) argumentan que la participación en el capital se puede volver más dispersa con cada transición generacional, ya que en una tercera generación, por ejemplo, hablamos de un consorcio de primos, donde habrá una dispersión de la propiedad entre ellos y posibles accionistas adicionales, por lo que la familia podría estar más alineada con inversionistas institucionales y accionistas externos (principalmente en empresas públicas), lo que conducirá a una mayor disposición a asumir riesgos y, por ende, hacia el uso la financiación a través de la deuda. En este sentido, Le Breton-Miller y Miller (2006), así como Gersick, Davis, Hampton y Lansberg (1997) resaltan la importancia de la relación a largo plazo entre las empresas familiares y los stakeholders externos, como los bancos principalmente, argumentando que si la transición de una empresa familiar se gestiona de forma adecuada, estas empresas pueden obtener mejores condiciones en la búsqueda de financiación a través de la deuda debido a que la relación de largo plazo entre banco y empresa puede generarse. De igual forma, establecen que las empresas familiares podrían tener mayores incentivos para cumplir con sus obligaciones, tanto en el corto como el largo plazo, ya que el nombre de la familia y su reputación estarían en juego.

Es importante destacar que esta relación entre sucesión y deuda se ha analizado a través de la relación que pudiese surgir entre las empresas familiares y los acreedores financieros una vez que la sucesión ha tenido lugar. En este sentido, autores como Blanco-Mazagatos et al. (2007) resaltan la creciente debilidad de los lazos familiares y los problemas de oportunismo que se pudiesen presentar en las empresas familiares a medida que se dan los diferentes cambios generacionales (empresas de tercera generación en adelante). De acuerdo con la teoría de la agencia, los miembros de la familia podrían tomar decisiones encaminadas al beneficio personal y no necesariamente hacia la creación de valor de la compañía (hablamos de imponerse sueldo y bonos excesivos, por ejemplo). De igual forma, miembros de la familia que no participen de forma activa en el negocio pueden buscar el uso de mayores niveles de financiación, ya que puede servir como mecanismo de gobierno para reducir los costos de agencia derivados de la discrecionalidad directiva que pudiese llegar a surgir (Gallo y Vilaseca, 1996; McConaughy, Matthews y Fialko, 2001). Así pues, se podría suponer que habrá una tendencia hacia la búsqueda de mayores niveles de endeudamiento por parte de las empresas familiares que se encuentren en manos de una tercera generación en adelante en comparación con aquellas empresas de segunda generación.

Como podemos apreciar, existe una clara relación entre la sucesión en empresas familiares y la estructura financiera de las mismas. Sin embargo, los resultados siguen siendo mixtos en este sentido; podemos encontrar literatura que argumenta una relación positiva entre sucesión y endeudamiento, así como también autores que encuentran una causalidad inversa. En este sentido, consideramos que estos resultados obtenidos son debido a los diferentes contextos en los que los estudios se han llevado a cabo, indudablemente aspectos como el entorno de negocios, protección legal a los inversionistas, niveles de concentración de propiedad, desarrollo de mercado, entre otros, son aspectos que desempeñan un papel fundamental a la hora de determinar el sentido de la relación. No obstante, es importante destacar que el efecto entre ambas variables puede ser diferente si hablamos de empresas que pasan del fundador a una segunda generación, que en aquellas empresas donde el cambio se produce de la segunda a la tercera generación o posteriores, debido a los mayores niveles de dispersión de la propiedad, tipos de inversionistas involucrados (sobre todo en empresas públicas), estructura de gobierno, etc. Por tanto, es importante también analizar si la relación entre sucesión y endeudamiento es diferente cuando nos referimos a empresas familiares que se encuentren en este nivel del proceso sucesorio en comparación con el obtenido en empresas de segunda generación. 


\section{Sucesión y desempeño}

Además de considerar la relación entre sucesión y deuda, es importante ver si la primera tiene un impacto en el desempeño de la compañía, ya que a final de cuentas el objetivo último de la empresa debe ser crear valor para los accionistas, y para ello la estructura financiera puede ejercer un papel fundamental. En este sentido, los trabajos que han analizado esta relación han llegado a resultados mixtos, ya que, por un lado, algunos autores argumentan que cuando el proceso de sucesión se ha llevado a cabo se genera una reducción en la creación de valor de la empresa, mientras que otros estudios han llegado a la relación opuesta. Muchos de los argumentos en este sentido son explicados principalmente por el hecho de considerar si dentro de los miembros de la familia de la siguiente generación existe en realidad la capacidad para generar mayor valor para la compañía. Lo anterior debido a que la alternativa de contratar a un directivo externo con una mayor preparación y experiencia regularmente suele ser ignorada, por lo que la empresa podría ver limitado, en cierta medida, su desempeño (Bennedsen et al., 2007; Cucculelli y Micucci, 2008). Autores como Amit y Villalonga (2006) encuentran una relación no monótona entre la generación que se encuentra al frente de la compañía y la creación de valor. Establecen que los directivos descendientes parecen ejercer un efecto negativo sobre el valor de la empresa, mientras que la relación se invierte en empresas de tercera generación. Por su parte Morck y Yeung (2003) señalan que podría esperarse un menor crecimiento en las empresas controladas por los descendientes, ya que estos podrían ser menos capaces en comparación con el fundador.

Por otra parte, algunos estudios se basan en la idea de que cuando las empresas familiares pasan de una generación a otra, sus objetivos cambian, lo que puede conducirlas hacia el estancamiento debido a que las empresas familiares de primera generación, por lo regular, se encuentran más orientadas hacia los negocios, mientras que aquellas que han pasado por un proceso de sucesión (segunda generación en adelante) se encuentran más orientadas hacia la familia, por lo que las primeras tienen una mayor capacidad para crecer y generar valor para la empresa (Ward, 1997; Cromie, Stevenson y Monteith, 1995; Dunn, 1995; Reid et al., 1999). En este sentido, Schulze et al. (2003) argumentan que empresas familiares que han evolucionado hacia una sociedad de hermanos (segunda generación) puede generar una mayor aversión al riesgo, llevando eventualmente a una reducción en el crecimiento de la compañía. Sin embargo, establecen que este efecto se invierte cuando se trata de una sociedad de primos (tercera generación), ya que en esta etapa la familia propietaria volverá a tener una mayor disposición al riesgo, centrándose más en el crecimiento. Martin y Lumpkin (2004) encuentran que en empresas donde se ha dado el cambio generacional, la orientación hacia el emprendimiento y creación de valor para la compañía tiende a disminuir, y se da mayor prioridad al aspecto familiar, por lo que cuestiones como la estabilidad y temas de herencia se vuelven prioritarios para las empresas. Por tanto, los autores apuntan a que esta mayor orientación hacia la familia puede limitar en cierta medida el desempeño, ya que hay una menor disposición al crecimiento y, por ende, hacia la generación de valor para la compañía. Davis y Harveston (1999) introdujeron el concepto de «sombra generacional», término que hace referencia a aquellos procesos de sucesión que consideran como «incompletos» debido a la continua influencia de los miembros de la familia de generaciones anteriores que ya no controlan directamente la empresa, por lo que este comportamiento puede limitar la motivación de los sucesores y aumentar la posibilidad de conflictos.

Por otro lado, autores como Zahra (2005) y Fernandez y Nieto (2005) establecen que cuando las nuevas generaciones participan de forma activa en la empresa, aumenta la riqueza de la misma debido a la renovación estratégica que se genera, ya que derivado del proceso de sucesión, los miembros de la familia de siguientes generaciones involucrados activamente en la empresa 
traerán nuevos conocimientos y puntos de vista diferentes y actuales que pueden impactar de forma positiva en aspectos como la innovación, internacionalización y, por ende, en el desempeño de la compañía. McConaughy y Phillips (1999), por su parte, también encuentran evidencia de que empresas familiares controladas por los descendientes son más rentables que aquellas empresas familiares controladas por el fundador, ya que aunque las empresas controladas por estos últimos tienen una mayor capacidad para crecer, las empresas dirigidas por descendientes tienen una mayor capacidad de generación de riqueza, debido a que pueden aprovechar las inversiones realizadas anteriormente por el fundador en bienes de capital y en $\mathrm{I}+\mathrm{D}$, por ejemplo.

Como se puede apreciar, estudios previos han encontrado evidencia de un cambio en el desempeño o performance de las empresas familiares después de que se ha llevado el proceso de transferencia generacional, además de mostrar que este efecto puede ser diferente entre sucesores de segunda generación y aquellos de tercera generación o posteriores.

\section{Hipótesis}

Basándonos en esta revisión de la literatura, planteamos 4 hipótesis que buscan contrastar la relación entre sucesión, endeudamiento y creación de valor, además de analizar si esta relación es diferente dependiendo del número de cambios generacionales que haya tenido la compañía (segunda o tercera generación en adelante). Sobre esta base proponemos las siguientes hipótesis:

Hipótesis 1. El proceso de sucesión (de primera a segunda generación) tiene un efecto sobre los niveles de endeudamiento de las empresas familiares.

Hipótesis 2. El proceso de sucesión de segunda a tercera generación tiene un efecto diferente sobre los niveles de endeudamiento de las empresas familiares, en comparación con el mostrado para empresas con un proceso de sucesión de primera a segunda generación.

Hipótesis 3. El proceso de sucesión (de primera a segunda generación) tiene un efecto sobre el desempeño (performance) de las empresas familiares.

Hipótesis 4. El proceso de sucesión de segunda a tercera generación tiene un efecto diferente sobre el desempeño (performance) de las empresas familiares, en comparación con el mostrado para empresas con un proceso de sucesión de primera a segunda generación.

\section{Metodología}

\section{Muestra}

En la muestra utilizada en este trabajo se toma como punto de partida aquellas empresas que cotizaron en la Bolsa mexicana de valores (BMV) durante los años 2005 y 2011. La fuente de información utilizada para la obtención de los estados financieros de las empresas es la base de datos «Economatica». La información relativa a la estructura de propiedad, cambios generacionales y gobierno de las compañías de la muestra utilizada en el análisis fue obtenida de los reportes anuales de las empresas publicados por la BMV en su página web ${ }^{1}$. De las 142 empresas que cotizan, fueron eliminadas aquellas sin fines de lucro, las que no contaban con suficiente información en sus estados financieros, así como las instituciones financieras. Estas

1 Véase http://www.bmv.com.mx. 
últimas se suprimieron debido a que no son comparables con otras industrias, además de presentar ciertas dificultades para calcular la Q de Tobin. De las 75 restantes se seleccionaron aquellas que pueden considerarse como empresas familiares. Para ello se siguieron 2 criterios: 1) que la propiedad por parte de una misma familia fuera al menos del $40 \%$; y 2) que se tuviera al menos un miembro de la familia propietaria en el gobierno y/o dirección de la empresa (San Martin-Reyna y Duran-Encalada, 2012). Las 56 empresas que componen la muestra final pueden considerarse representativas del tejido empresarial mexicano debido a los distintos sectores que en dicha muestra se encuentran. La tabla 1 muestra las empresas que conforman la muestra, de acuerdo a los sectores a los que pertenecen y cambios generacionales a los que se han sometido.

Como podemos observar, las empresas que componen la muestra son medianas y grandes básicamente. Esto podría plantear algunas advertencias sobre un posible sesgo en la muestra, no obstante, el tamaño de la empresa (en términos de activos) es bastante heterogéneo y muy disperso en torno al valor medio, por lo que se asume que los resultados no están sesgados por problemas de tamaño. Es importante destacar el hecho de que las empresas de primera generación son las más grandes (en términos de activos), seguidas por aquellas empresas que se encuentran en segunda generación, siendo las más pequeñas las de tercera generación. Lo anterior llama la atención debido a que se esperaría que las empresas que han logrado pasar a las manos de varias generaciones hayan ido generando una mayor cantidad de activos. Se puede explicar esto por el hecho de que el promedio de activos de las empresas de primera generación se encuentra altamente influenciado por el valor extremo que representan los activos de América Móvil. Por otra parte, podemos destacar que la composición de la muestra es bastante equilibrada, aunque hay un ligero sesgo hacia las empresas industriales y de materiales a expensas de las empresas de salud o de telecomunicaciones. Esta composición por sectores refleja la composición de la estructura empresarial mexicana.

\section{Modelo de regresión y definición de variables}

El objetivo del análisis empírico propuesto es contrastar la asociación entre la sucesión, la deuda y la creación de valor, contemplando a la vez si el efecto es diferente dependiendo de en qué cambio generacional se encuentra la compañía. Dado el objetivo del estudio, la metodología de datos de panel parece ser la más apropiada, ya que tal y como señalan Molly et al. (2010) la mayoría de los estudios hasta ahora se han centrado en analizar estos efectos de cambios generacionales basados en datos de corte transversal, lo que tiene una serie de limitaciones, ya que en primera instancia este tipo de datos no permite incorporar al modelo análisis longitudinales que pudiesen afectar el desempeño financiero, así como la estructura de capital de la compañía, ya que analizan la empresa a un año solamente de haberse llevado a cabo la sucesión. Es por ello que en este trabajo llevamos a cabo ese análisis longitudinal tratando de identificar con mayor precisión los efectos que la sucesión puede tener dentro de la empresa familiar. Además, el término de efectos fijos no es observable y, por tanto, se convierte en parte del componente aleatorio en el modelo estimado. Si no tomamos esto en cuenta y llevamos a cabo un análisis a través de mínimos cuadrados ordinarios, por ejemplo, podría causar un sesgo de omisión y distorsionar los resultados. Por ello debemos tener en cuenta que la variación en la estructura de capital y desempeño, una vez que la sucesión ha tenido lugar, implica controlar también aquellas características invariantes en el tiempo y que muchas veces son difíciles de observar o medir (la historia o la cultura de la empresa familiar, por ejemplo). Estas características varían a través de las empresas, pero si no lo tenemos en cuenta podríamos asumir que son constantes en cada una de estas (Molly et al., 
Tabla 1

Número y porcentaje de empresas por sector de acuerdo a la transferencia generacional

\begin{tabular}{|c|c|c|c|c|c|c|}
\hline \multirow[b]{2}{*}{ Características } & \multicolumn{2}{|c|}{$\begin{array}{l}\text { Empresas familiares } \\
\text { transferidas de la primera a la } \\
\text { segunda generación }\end{array}$} & \multicolumn{2}{|c|}{$\begin{array}{c}\text { Empresas familiares } \\
\text { transferidas de una segunda a } \\
\text { una tercera generación }\end{array}$} & \multicolumn{2}{|c|}{$\begin{array}{c}\text { Empresas de primera } \\
\text { generación }\end{array}$} \\
\hline & $\mathrm{N}$ & $\%$ & $\mathrm{~N}$ & $\%$ & $\mathrm{~N}$ & $\%$ \\
\hline Número de empresas & 23 & & 12 & & 21 & \\
\hline \multicolumn{7}{|l|}{ Sector y Proporción } \\
\hline Materiales & 4 & 17.3 & 1 & 8.3 & 6 & 28.57 \\
\hline Industrial & 5 & 21.7 & 4 & 33.33 & 6 & 28.57 \\
\hline Servicio y bienes de consumo no básicos & 5 & 21.7 & 3 & 25 & 3 & 14.28 \\
\hline Productos de consumo frecuente & 4 & 17.3 & 3 & 25 & 4 & 19 \\
\hline Salud & 2 & 8.6 & 1 & 8.3 & 0 & \\
\hline Telecomunicaciones & 3 & 13 & 0 & & 2 & 9.5 \\
\hline Edad de la empresa (promedio) & 32 & & 54.7 & & 42 & \\
\hline Total activos (promedio) & 47032.7 & & 24175.7 & & 1030941.0 & \\
\hline
\end{tabular}

Fuente: Códigos de clasificación de la Bolsa Mexicana de Valores, 2013. 
2010), por lo que el análisis de efectos fijos permite controlar esta heterogeneidad inobservable. Así pues, los modelos se pueden expresar de la siguiente manera, donde i se refiere a las empresas y t al año $(i=1 \ldots 75 ; t=1 \ldots 7)$

$$
\begin{aligned}
\text { Endeudamiento }_{i t} & =\beta_{i t}+\text { Ptrans }_{i t}+\text { Stransf }_{i t}+\text { lAt }_{i t}+\text { Colaterales }_{i t} \\
& + \text { Retornoactivos }_{i t}+\text { Crecactivos }_{i t}+\varepsilon_{i t}
\end{aligned}
$$$$
{\text { Qde } \text { Tobin }_{i t}}=\beta_{i t}+\text { Ptrans }_{i t}+\text { Strans }_{i t}+\text { lAt }_{i t}+\text { Crecactivos }_{i t}+\varepsilon_{i t}
$$

Donde: Endeudamiento: apalancamiento de la empresa, medido por el pasivo total entre el activo total. Q de Tobin: está determinada por el valor de mercado de los activos más valor en libros de la deuda sobre los activos totales. Ptransf: variable dummy que toma valor de 1 si la sucesión fue de primera a segunda generación y 0 en otro caso. Stransf: Variable dummy que toma el valor de 1 si la sucesión fue de segunda a tercera generación en adelante y 0 en otro caso. 1At: logaritmo del activo total. Colaterales: calculado como activos fijos entre activos totales. Retornoactivos: Utilidad antes de intereses, impuestos, depreciación y amortización entre activo total. Crecactivos: Tasa de crecimiento en activos.

Las variables dependientes son endeudamiento y desempeño (performance) o creación de valor estimada a través de la Q de Tobin, ya que nuestro interés es analizar si existe relación entre diferentes etapas del proceso de sucesión y la estructura financiera y de capital de la empresa. Las variables independientes están relacionadas con el proceso de sucesión, donde Pransf se refiere a la primera transferencia dentro de la empresa familiar y representa el cambio de primera a segunda generación, mientras que Stransf representa el cambio generacional de segunda a tercera generación (para nuestro caso en particular solo es de segunda a tercera, ya que en la muestra no se obtuvieron empresas que se encuentren en cuarta generación o posteriores). Ambas variables son dummis que toman el valor de 1 en el año posterior a la sucesión y 0 en otro caso. Con respecto a las variables de control, y en función de lo realizado en trabajos previos (Perez-Gonzalez, 2006; Huson, Malatesta y Parrino 2004; Denis y Denis, 1995; Anderson y Reeb, 2003; Sraer y Thesmar, 2007; Romano, Tanewski y Smyrnios, 2001; Delmar, Davidsson y Gartner, 2003; Honjo y Harada, 2006; Molly et al., 2010) hemos incluido la variable colaterales, que se utiliza como una medida del valor de la garantía de la empresa. Podría suponerse que cuanto mayores sean los activos de la empresa mayor probabilidad de financiación por parte de los bancos habrá (Harris y Raviv, 1991; Rajan y Zingales, 1995; Fama y French, 2002). También incluimos la variable retorno de los activos, ya que esta variable es una medida de la capacidad de financiación interna de la empresa, por lo que podría esperarse un relación negativa con la deuda, ya que muchos propietarios y directivos (sobre todo de carácter familiar) preferirán financiar sus actividades con fondos de la propia empresa en lugar de recurrir a la deuda (Fernández y Gómez, 1999). Con respecto a la variable crecimiento en activos, esta se incluye debido a que de acuerdo con Carpenter y Petersen (2002) y Molly et al. (2010), la tasa de crecimiento de activos de la empresa puede verse limitada por la disponibilidad de recursos financieros internos. Finalmente, incluimos también el tamaño de la empresa como variable de control.

\section{Resultados}

Los resultados de la estimación se muestran en las tablas 2 y 3. La tabla 2 hace referencia a la estimación para analizar los efectos de la sucesión en la estructura de capital de las empresas familiares, mientras que la tabla 3 muestra estos efectos sobre el desempeño financiero de las 
Tabla 2

Resultados de la estimación sobre endeudamiento

\begin{tabular}{|c|c|c|c|}
\hline Variables & Coeficiente & Estadístico t & Valor de $\mathrm{p}$ \\
\hline Ptransf & -0.143 & -2.26 & {$[0.024]^{*}$} \\
\hline Stransf & 0.109 & 3.16 & {$[0.002]^{* *}$} \\
\hline 1At & 0.026 & 2.07 & {$[0.039]^{*}$} \\
\hline Colaterales & -0.006 & -2.23 & {$[0.027]^{*}$} \\
\hline Retornoactivos & -0.007 & -0.11 & [0.909] \\
\hline Crecactivos & 0.001 & 1.87 & {$[0.063]^{* * *}$} \\
\hline Constante & 0.567 & 4.71 & 0.000 \\
\hline $\mathrm{R}^{2}$ ajustada & 0.21 & & \\
\hline Test de Hausman & 40.95 & & 0.000 \\
\hline
\end{tabular}

Endeudamiento es la variable dependiente y está determinada por el pasivo total entre el activo total. Pransf es el cambio de primera a segunda generación en la empresa y Stransf es el cambio generacional de segunda generación en adelante. Ambas variables son dummis que toman el valor de 1 en el año posterior a la sucesión y 0 en otro caso. 1 At es el logaritmo del activo total, y es la proxy del tamaño de la empresa. Colaterales es la relación entre activos fijos y activos totales, Retornoactivos se determinan como las ganancias antes de intereses, impuestos, depreciación y amortización entre activo total. Crecactivos es la tasa de crecimiento de los activos. El test de Hausman permite probar la hipótesis efectos fijos vs efectos aleatorios en la estimación. El test de Hausman sigue una distribución Chi cuadrado.

La tabla muestra los coeficientes estimados, el t estadístico y el valor de p.

* Significativo a 0.05 .

*** Significativo a 0.01 .

*** Significativo a 0.10 .

Tabla 3

Resultados de la estimación sobre desempeño (Q de Tobin)

\begin{tabular}{lccc}
\hline Variables & Coeficiente & Estadístico t & Valor de $\mathrm{p}$ \\
\hline Ptransf & -0.273 & -0.57 & {$[0.572]$} \\
Stransf & 0.499 & 1.89 & {$[0.056]^{*}$} \\
At & 0.351 & 2.83 & {$[0.005]^{* *}$} \\
Crecactivos & 0.068 & 1.54 & {$[0.125]$} \\
Constante & 0.937 & 1.37 & {$[0.171]$} \\
$\mathrm{R}^{2}$ ajustada & 0.23 & & 0.000 \\
Test de Hausman & 33.71 & &
\end{tabular}

La Q de Tobin es la variable dependiente y está determinada por el valor de mercado de los activos más valor en libros de la deuda sobre los activos totales. Pransf es el cambio de primera a segunda generación en la empresa y Stransf es el cambio generacional de segunda generación en adelante (para nuestro caso en particular solo es de segunda a tercera, ya que en la muestra no se obtuvieron empresas de cuarta generación en adelante). Ambas variables son dummis que toman el valor de 1 en el año posterior a la sucesión y 0 en otro caso. 1At es el logaritmo del activo total, y es la proxy del tamaño de la empresa. Crecactivos es la tasa de crecimiento de los activos. El test de Hausman permite probar la hipótesis efectos fijos vs efectos aleatorios en la estimación. El test de Hausman sigue una distribución Chi cuadrado.

La tabla muestral los coeficientes estimados, el t estadístico y el valor de $\mathrm{p}$.

* Significativo a 0.10 .

*** Significativo a 0.01 .

compañías. La prueba de Hausman muestra la importancia del componente de efectos fijos, por lo que dentro del método de estimación es necesario realizar las estimaciones de esta manera con el fin de hacer frente a la heterogeneidad inobservable.

Como podemos apreciar en la tabla 2, los resultados muestran un efecto diferente de la sucesión sobre los niveles de deuda adquiridos por la compañía dependiendo de la generación al frente de la empresa. Es decir, encontramos una relación negativa y significativa entre la transferencia 
de la empresa de la primera a la segunda generación sobre el endeudamiento, lo que nos deja ver que estas empresas tienen una mayor aversión al riesgo, prevaleciendo la idea de asegurar el futuro de la compañía, así como la reputación y nombre de la familia, buscando legar una empresa sana a sus descendientes (efecto estancamiento). Mientras que, por otro lado, aquellas empresas que han logrado superar este primer paso generacional y se encuentran en una tercera generación muestran una mayor disposición al riesgo, adquiriendo mayores niveles de endeudamiento con el fin de revitalizar la compañía. Hablamos de empresas en las que existe una sociedad de primos, donde los nietos de los fundadores están incorporados a la compañía y la propiedad, aunque siga siendo familia, está mucho más dispersa con nuevas generaciones y accionistas tratando de generar mayor valor para la empresa. De esta forma, las hipótesis 1 y 2 quedan comprobadas al mostrar que dependiendo del cambio generacional al que se haya sometido la empresa familiar, la relación con los niveles de endeudamiento de la compañía es diferente. Con respecto al resto de las variables, tamaño y crecimiento en activos tienen una relación positiva con el endeudamiento. Es decir, cuanto más grandes son las empresas mayores niveles de endeudamiento tienen, siendo también el crecimiento de la empresa un factor relevante para adquirir mayor financiación. Por otro lado, los colaterales y el retorno de los activos tienen una relación negativa con la deuda. La primera de ella presenta un resultado opuesto al esperado, ya que podría suponerse que cuanto mayores sean los colaterales que una empresa muestra, mayores posibilidades de financiación obtendrá. Los resultados obtenidos pueden ser explicados en el sentido de que los activos de la empresa son relativamente líquidos, por lo que no ayudan en gran medida como colateral para la obtención de financiación o puede ser que tengan una mayor proporción de deuda de corto plazo, por lo que la empresa podría estar alineando sus activos con el tipo de pasivos adquiridos (Molly et al., 2010). Por último, el retorno de los activos muestra el resultado esperado, ya que cuanto mayores sean los recursos generados por la propia compañía, menores serán las necesidades de financiación a través de deuda, aunque se reconoce que este resultado no es estadísticamente significativo.

Tratando de ir un poco más allá en los resultados, analizamos si esta relación que encontramos entre sucesión y estructura financiera en realidad tiene un impacto sobre la creación de valor; es decir, tal vez las segundas generaciones se dejen llevar por esa actitud que podemos denominar «conservadora», ya que en esa etapa de la compañía podría ser lo adecuado para generar valor para la misma, y esta actitud de menor aversión al riesgo en terceras generaciones al atraer mayor financiación para la compañía en realidad podría no verse plasmada en la creación de valor. Por ello, es que llevamos a cabo un segundo modelo donde tratamos de identificar si el paso generacional tiene algún efecto sobre la creación de valor y, de ser así, si ese impacto es diferente dependiendo del cambio generacional que haya enfrentado la empresa familiar. Los resultados se pueden apreciar en la tabla 3.

La tabla 3 muestra los resultados respecto al análisis de la relación entre sucesión y creación de valor. Como podemos apreciar, los resultados obtenidos muestran que el cambio de la primera a la segunda generación no tiene un efecto significativo en la creación de valor, lo que podríamos atribuir a la actitud mucho más conservadora de esta generación al frente de la empresa. Sin embargo, cuando analizamos los efectos de la transición a la tercera generación, los efectos sobre la creación de valor son positivos y significativos, indicando que el mayor uso de la deuda en realidad sí está asociado a una mayor creación de valor para la compañía, con lo que podemos confirmar la hipótesis 4 , mientras que la 3 no se ha comprobado. Por lo que respecta al resto de las variables, solo el tamaño tiene un efecto positivo y significativo sobre la creación de valor.

De esta forma, se puede apreciar que el negocio familiar tiene niveles de apalancamiento y desempeño diferentes no solo derivados del proceso de sucesión, sino también del número de 
cambios generacionales que han tenido lugar dentro de la compañía, ya que los resultados obtenidos muestran que la transición entre el fundador y la segunda generación puede ser considerada como la menos arriesgada (en términos de endeudamiento) y también como la que tiende a generar un mayor estancamiento de la compañía, mientras que el efecto significativo de la sucesión en el desempeño financiero y la estructura de capital de la empresa se produce principalmente en aquellas empresas familiares cuyo proceso de sucesión se ha dado entre la segunda y tercera generación.

\section{Conclusiones}

Cuando hablamos de empresas familiares, sin lugar a dudas hacemos referencia a un tipo de empresa que tiene que ser analizada de forma diferente. Hablamos de entidades que no sufren solo de los problemas característicos que pudiesen tener las empresas, ya que además de ello tenemos que agregar un ingrediente más que es la familia. Ese traslape entre empresa y familia es lo que ha detonado un gran número de trabajos que tratan de entender y explicar comportamientos y funcionamientos de este tipo de compañías. Dentro de todo este mar de líneas de investigación que han surgido derivadas de las empresas familiares, la sucesión es una de las predilectas por representar todo un reto para estas empresas. Desafortunadamente, es la minoría la que logra superar este trance generacional, legar la empresa a los descendientes, lo que resulta paradójico, ya que muchas veces es el fin para el que se crearon. Por si fuera poco, el tema se vuelve trascendental, sobre todo si consideramos que muchas de las empresas en el ambiente de negocios mexicano se aproximan en los años venideros a un cambio generacional en la compañía, donde aspectos como estructuras financieras y creación de valor son piezas clave dentro del rompecabezas que implica el tránsito generacional. Es por ello que consideramos importante analizar y entender la sucesión, ya que al hacerlo podemos generar mayor conocimiento sobre mejores prácticas corporativas y entender por qué la empresa cambia como resultado del efecto de la sucesión.

Nuestros resultados muestran que el proceso de sucesión de la primera a la segunda generación tiene un efecto negativo en los niveles de endeudamiento de la empresa, lo que puede dar lugar a algún tipo de estancamiento con una estructura financiera más conservadora y una limitación de la tasa de crecimiento de la compañía. Sin embargo, cuando se considera el proceso del cambio generacional de una segunda a tercera generación el resultado se invierte, es decir, las empresas familiares en manos de la tercera generación tienen una menor aversión al riesgo y tienen mayor propensión al uso de la deuda. Por lo que respecta al efecto que el cambio generacional tiene sobre la creación de valor, nuestros resultados muestran que el cambio entre primera y segunda generación no tiene ningún efecto sobre la creación de valor, sin embargo cuando se considera el cambio de una segunda a tercera generación esta transición sí tiene efecto positivo y significativo sobre el desempeño o performance de la compañía.

Como podemos apreciar, nuestros resultados muestran que las empresas familiares a menudo se vuelven más reacias al riesgo, sobre todo después del primer cambio generacional. Los miembros de la próxima generación parecen mostrar una mayor preocupación por la preservación de la riqueza que por el incremento de la misma, lo que puede dar lugar a niveles de endeudamiento bajos con una mayor orientación hacia la búsqueda de crecimientos moderados pero menos arriesgados, mientras que empresas en manos de una tercera generación se caracterizan por una menor aversión al riesgo, por lo que son más agresivos a la hora de buscar tasas de crecimiento.

Muy posiblemente esto también pueda explicarse por factores que se asocian al perfil familiar o no del sucesor en el cargo de la dirección y/o presidencia del consejo de administración de la empresa. Es más probable que en la trasferencia de una primera a una segunda generación el 
gobierno corporativo y la dirección de la empresa caiga en un miembro familiar, lo cual contribuye a una mayor aversión al riesgo y a un mayor contenido de objetivos socioemocionales en la conducción de la empresa (Gómez-Mejía, Haynes, Núñez-Nickel, Jacobson y Moyano-Fuentes, 2007). En tanto que al pasar a una tercera generación, la dispersión misma de propiedad entre diferentes miembros o ramas familiares hace necesaria una mayor participación de miembros nofamiliares en la dirección y gobierno de la empresa, adquiriendo un enfoque más dirigido hacia la creación de valor económico en la empresa y los riesgos que ello conlleva (Miller, Minichilli y Corbetta, 2013).

Desde una perspectiva práctica, la diferencia observada en el comportamiento de los niveles de deuda entre las generaciones puede servir para llenar los vacíos institucionales en países emergentes como México, tal como señala Gedajlovic, Carney, Chrisman y Kellermanns (2012), principalmente la débil protección proporcionada a accionistas minoritarios. En este contexto, el efecto reforzador de tener un bajo endeudamiento puede contribuir a una asociación de la empresa familiar con un mejor desempeño. Sin embargo, el desempeño relativo más bajo que es aparente entre el obtenido en la transición de una primera a una segunda generación, comparado con el de la segunda a una tercera generación, puede ser resultado de decisiones corporativas en relación con el endeudamiento, que en ciertos casos puede llevar a una subinversión que de otra manera podría apoyar el crecimiento y rentabilidad de la empresa. Por lo tanto, con el propósito de hacer mejor uso de su potencial, se recomienda que las empresas familiares, sobre todo en una segunda generación, creen estructuras organizacionales y reglas de decisión que faciliten la comunicación intraorganizacional, incluyendo el involucramiento de miembros familiares en la alta dirección de las empresa, donde la participación de consultores profesionales puede también contribuir (De Massis, Kotlar, Campopiano y Cassia, 2013).

En cuanto a las limitaciones de nuestro estudio, es importante señalar que el tema de la sucesión es algo muy complejo y que puede ser abordado desde diferentes vertientes, algunas de las cuales escapan a este trabajo, aspectos como conflictos intrafamiliares durante el proceso de sucesión (De Massis et al., 2008), preparación y selección adecuada del sucesor (Le Breton-Miller, Miller y Steier, 2004; Sharma y Irving, 2005; Brun de Pontet, Wrosch y Gagne, 2007), planificación de la sucesión (Cabrera-Suárez y Martín Santana, 2006), relevancia del conocimiento general y técnico de la industria (Royer, Simons, Boyd y Rafferty, 2008) y la ya mencionada sobre la pertenencia o no del sucesor a la familia (Miller et al., 2013), entre otros, son aspectos que no se consideran dentro de nuestro trabajo. En este partimos del hecho de que el proceso de sucesión se llevó a cabo, y es posible que el proceso en el que la sucesión se produjo pudiese afectar la forma en que las nuevas generaciones hacen frente a aspectos como endeudamiento y creación de valor para la compañía. Por tanto, sería interesante como futura línea de investigación contrastar nuestros resultados a través de métodos cualitativos (como son las entrevistas en profundidad y los análisis psicosociales), lo que permitiría acercar los resultados aún más a las empresas familiares y darles una mayor practicidad a los mismos, indagando en el por qué las empresas familiares de alguna manera enseñan a sus hijos a alejarse del riesgo y a orientarse más en preservar la riqueza heredada, dejando de lado el alentar el espíritu emprendedor por parte de la segunda generación, por lo que aspectos cualitativos como estos podrían darnos unos resultados con mayor robustez.

\section{Referencias}

Amit, R. y Villalonga, B. (2006). How do family ownership, control and management affect firm value? Journal of Financial Economics, 80, 385-417. 
Anderson, R. C., Mansi, A. S. y Reeb, D. M. (2003). Founding family ownership and the agency cost of debt. Journal of Financial Economics, 68, 263-285.

Bennedsen, M., Nielsen, K., Perez-Gonzalez, F. y Wolfenzon, D. (2007). Inside the family firm: The role of families in succession decisions and performance. Quarterly Journal of Economics, 122, 647-691.

Birley, S. (1986). Succession in the family firm: The inheritor's view. Journal of Small Business Management, 24, $36-43$.

Blanco-Mazagatos, V., de Quevedo-Puente, E. y Castrillo, L. A. (2007). The trade-off between financial resources and agency costs in the family business: An exploratory study. Family Business Review, 20, 199-213.

Brun de Pontet, S., Wrosch, C. y Gagne, M. (2007). An exploration of the generational differences in levels of control held among family business approaching succession. Family Business Review, 20, 337-354.

Cabrera-Suárez, M. K. y Martín Santana, J. D. (2006). Factores que afectan al proceso de sucesión en la empresa familiar: Perspectiva del predecesor versus sucesor. Conocimiento, innovación y emprendedores: Camino al futuro, $1258-1275$.

Castrillo, L. A. y San-Martín, J. M. (2007). La propiedad familiar como mecanismo de gobierno disciplinador de la dirección en las empresas mexicanas: Una evidencia empírica. Revista Contaduría y Administración, 222, 59-82.

Casson, M. (1999). The Economics of the Family Firm. Scandinavian Economic History Review, 47, 10-23.

Carpenter, R. E. y Petersen, B. C. (2002). Is the growth of small firms constrained by internal finance? Review of Economics and Statistics, 84, 298-309.

Chami R. (1999). What's different about family business? Working paper. Trabajo publicado en la serie del Fondo Monetario Internacional, número 01/70.

Cromie, S., Stevenson, B. y Monteith, D. (1995). The management of family firms: An empirical investigation. International Small Business Journal, 13, 11-34.

Cucculelli, M. y Micucci, G. (2008). Family succession and firm performance: Evidence from Italian family firms. Journal of Corporate Finance, 14, 17-31.

Dunn, B. (1995). Success themes in Scottish family enterprises: Philosophies and practices through generations. Family Business Review, 8, 17-28.

Davis, P. S. y Harveston, P. D. (1999). In the founder's shadow: Conflict in the family firm. Family Business Review, 12 , 311-323.

Daily, C. M. y Dollinger, M. J. (1992). An empirical examination of ownership structure in family and professionally managed firms. Family Business Review, 5, 117-136.

De Massis, A., Chua, J. H. y Chrisman, J. J. (2008). Factors preventing intra-family succession. Family Business Review, 21, 183-199.

De Massis, A., Kotlar, J., Campopiano, G. y Cassia, L. (2013). Dispersion of family ownership and the performance of small-to-medium size private family firms. Journal of Family Business Strategy, 4, 166-175.

Delmar, F., Davidsson, P. y Gartner, W. B. (2003). Arriving at the high-growth firm. Journal of Business Venturing, 18, $189-216$.

Denis, D. J. y Denis, D. K. (1995). Performance changes following top management dismissals. Journal of Finance, 50, 1029-1057.

Fama, E. F. y Jensen, M. C. (1983). Agency problems and residual claims. Journal of Law and Economics, 26, 327-349.

Fama, E. F. y French, K. R. (2002). Testing trade-off and pecking order predictions about dividends and debt. Review of Financial Studies, 15, 1-33.

Fernández, A. I. y Gómez, S. (1999). Un estudio de las ofertas públicas de adquisición en el mercado de capitales español. Investigaciones Económicas, 23, 471-495.

Fernandez, Z. y Nieto, M. J. (2005). Internationalization strategy of small and medium-sized family businesses: Some influential factors. Family Business Review, 18, 77-89.

Gallo, M. y Vilaseca, A. (1996). Finance in family business. Family Business Review, 9, 287-305.

Gedajlovic, E., Carney, M., Chrisman, J. y Kellermanns, F. (2012). The Adolescence of family firm research: Taking stock and planning for the future. Journal of Management, 38, 1010-1037.

Gersick, K., Davis, J., Hampton, M. y Lansberg, I. (1997). Generation to generation: Life cycles of the family business. Boston: Harvard Business School Press.

Gómez-Mejía, L. R., Haynes, K. T., Núñez-Nickel, M., Jacobson, K. J. L. y Moyano-Fuentes, J. (2007). Socioemotional wealth and business risks in family-controlled firms: Evidence from Spanish olive oil mills. Administrative Science Quarterly, 52(1), 106-137.

Harris, M. y Raviv, A. (1991). The theory of capital structure. Journal of Finance, 46, 297-355.

Honjo, Y. y Harada, N. (2006). SME policy, financial structure and firm growth: Evidence from Japan. Small Business Economics, 27, 289-300. 
Huson, M. R., Malatesta, P. H. y Parrino, R. (2004). Managerial succession and firm performance. Journal of Financial Economics, 74, 237-275.

Jensen, M. C. y Meckling, W. (1976). Theory of the firm: Managerial behavior, agency costs and ownership structure. Journal of Financial Economics, 3, 305-360.

Kaye, K. y Hamilton, S. (2004). Roles of trust in consulting to financial families. Family Business Review, 17, $151-163$.

Kets de Vries, M. (1993). The dynamics of family-controlled firms: The good and bad news. Organisational Dynamics, 21, 59-68.

Le Breton-Miller, I., Miller, D., Steier, L.P. (2004). Toward an integrative model of effective FOB sucesión. Entrepreneurship Theory and Practice, 28, 305-328.

Le Breton-Miller, I. y Miller, D. (2006). Why do some family businesses out-compete? Governance, long-term orientations, and sustainable capability. Entrepreneurship Theory and Practice, 30, 731-746.

Martin, W. L. y Lumpkin, G. T. (2004). From entrepreneurial orientation to family orientation: Generational differences in the management of family businesses. Frontiers of entrepreneurship research. Wellesley, MA: Babson College.

McConaughy, D. L. y Phillips, G. M. (1999). Founders versus descendants: The profitability, efficiency, growth characteristics and financing in large, public founding-family controlled firms. Family Business Review, 12, $123-131$.

McConaughy, D. L., Matthews, C. H. y Fialko, A. S. (2001). Founding family controlled firms: Performance, risk and value. Journal of Small Business Management, 39, 31-49.

Miller, D. y Le Breton-Miller, I. (2005). Managing for the long run: Lessons in competitive advantage from great family businesses. Boston: Harvard Business School Press.

Miller, D., Le Breton-Miller, I. y Scholnick, B. (2008). Stewardship vs. stagnation: An empirical comparison of small family and non-family businesses. Journal of Management Studies, 45, 51-78.

Miller, D., Minichilli, A. y Corbetta, G. (2013). Is family leadership always beneficial? Strategic Management Journal, 34, 553-571.

Molly, V., Leveren, E. y Deloof, M. (2010). Family business succession and its impact on financial structure and performance. Family Business Review, 23, 131-147.

Morck, R. y Yeung, B. (2003). Agency problems in large family business groups. Entrepreneurship Theory and Practice, 27, 367-382.

Paul, J. J. (1996). Family business survival. Blueprint for Business Success, 16, 1-5.

Perez-Gonzalez, F. (2006). Inherited control and firm performance. American Economic Review, 96, 1559-1588.

Rajan, R. G. y Zingales, L. (1995). What do we know about capital structure? Some evidence from international data. Journal of Finance, 50, 1421-1460.

Reid, R., Dunn, B., Cromie, S. y Adams, J. (1999). Family orientation in family firms: A model and some empirical evidence. Journal of Small Business and Enterprise Development, 6, 55-66.

Romano, C. A., Tanewski, G. A. y Smyrnios, K. X. (2001). Capital structure decision making: A model for family business. Journal of Business Venturing, 16, 285-310.

Royer, S., Simons, R., Boyd, B. y Rafferty, A. (2008). Promoting family: A contingency model of family business succession. Family Business Review, 21, 15-30.

San Martin-Reyna, J. M. y Duran-Encalada, J. A. (2012). Relationship among family business, corporate governance and firm performance: Evidence from the Mexican stock exchange. Journal of Family Business Strategy, 3 , $106-117$.

Schulze, W. S., Lubatkin, M. H. y Dino, R. N. (2003). Exploring the agency consequences of ownership dispersion among the directors of private family firms. Academy of Management Journal, 46, 179-194.

Schwass, J. (2005). Wise growth strategies in leading family businesses. New York: Palgrave Macmillan.

Secretaría de Economía (2014). Presenta la Secretaría de economía y la Bolsa mexicana de valores programa para Pymes [consultado 10 Abr 2014]. Disponible en: http://www.economia.gob.mx/eventos-noticias/ sala-de-prensa/comunicados/6556-presentan-la-secretara-de-economa-y-la-bolsa-mexicana-de-valores-programapara-pymes

Sharma, P. y Irving, G. P. (2005). Four bases of family business successor commitment: Antecedents and consequences. Entrepreneurship Theory and Practice, 29, 13-33.

Shleifer, A. y Vishny, R. W. (1986). Large shareholders and corporate control. Journal of Political Economy, 94, 461-488.

Smith, B. F. y Amoako-Adu, B. (1999). Management succession and financial performance of family controlled firms. Journal of Corporate Finance, 5, 341-368.

Sraer, D. y Thesmar, D. (2007). Performance and behavior of family firms: Evidence from the French stock market. Journal of the European Economic Association, 5, 709-751.

Trevinyo-Rodríguez, R. N. (2010). Empresas familiares. Visión latinoamericana. Estructura, gestión, crecimiento y continuidad. México: Pearson Educación. 
Ward, J. L. (1997). Keeping the family business healthy: How to plan for continuing growth, profitability and family leadership. Marietta, GA: Business Owner Resources.

Zahra, S. A. (2005). Entrepreneurial risk taking in family firms. Family Business Review, 18, $23-40$.

Zúñiga, J. A. y Sacristán, M. (2009). Los directivos externos y la sucesión en la empresa familiar: un caso de estudio. Universia Business Review, 22, 74-87. 